Arch. math. Logik 22 (1982), 103-120

\title{
EXISTENTIAL INTERPRETATION. II *
}

\author{
Yuri Gurevich
}

\begin{abstract}
A method of existential interpretation was introduced in [2]. It allows proving undecidability of modest strata of many first order theories. Here we improve the method and its presentation, strengthen somewhat the previous results and prove a couple of new results. The reader is not supposed to be acquainted with [2].
\end{abstract}

\section{Introduction}

Undecidability of a first order theory $T$ is proved often by interpreting another first order theory $T^{\prime}$, known to be undecidable, in $T$. Suppose for simplicity that $T^{\prime}$ is a theory of a binary predicate $P$ and each formula $\varphi$ in the language of $T^{\prime}$ is translated into the language of $T$ by replacing $P$ by a formula $\pi$ with two free variables. Suppose that both $\pi$ and its negation are equivalent in $T$ to existential formulas. Then the translation of an $\forall \forall^{P} \exists \ldots \exists$ prenex sentence $\varphi$ is equivalent in $T$ to an $\forall^{p} \exists \ldots \exists$ prenex sentence. That reduces the decision problem for $\forall^{p} \exists \ldots \exists$ sentences of $T^{\prime}$ to the corresponding problem of $T$. That simple note gives an idea of the method of existential interpretation introduced in [2].

Undecidability of modest strata of some first order algebraic theories was proved in [2]. Among those theories are the theory of partial order, the theory of lattices, the theories of different classes of metabelian groups and so on. Many additional results of that sort can be obtained by the existential interpretation method. The theory of one irreflexive symmetrical predicate is especially convenient to be interpreted in other theories. Call it ISP. According to [2], (i) the set of $\forall^{3} \exists \ldots \exists$ sentences satisfiable in models of ISP is decidable, and (ii) the set of $\forall^{6} \exists \ldots \exists$ sentences not satisfiable in models of ISP and the set of $\forall^{6} \exists \ldots \exists$ sentences satisfiable in finite models of ISP are recursively inseparable. Here we prove that the set of logically false $\forall^{5} \exists \ldots \exists$ sentences in the language of ISP and the set of $\forall^{5} \exists \ldots \exists$ sentences satisfiable in finite models of ISP are effectively inseparable. In particular that improvement implies improvements in the results of [2] related to group theory. The decision problem for $\forall^{4} \exists \ldots \exists$ stratum of ISP remains open. The results of [2] were reported in 1965 in Malcev's logic seminar in Novosibirsk.

* Eingegangen am 26.11.1979. 
One of the listeners, Yuri Ershov, expressed an opinion that the existential interpretation method would not work for more sophisticated theories and in particular for the theory of two equivalence relations. Call the latter theory TER. Eventually the method does work for TER. It is proved below that the set of logically false $\forall^{5} \exists \ldots \exists$ sentences in the language of TER and the set of $\forall^{5} \exists \ldots \exists$ sentences satisfiable in finite models of TER are effectively inseparable. The inseparability results for ISP and TER were announced in [3].

An important aim of this paper is a better exposition of the existential interpretation method. The reader isn't supposed to be familiar with [2]. Section 1 is the main part of the exposition. The presentation there isn't in most general form. It gives enough to deal with the theories mentioned above and, we hope, it is easily readable. A number of generalizations are more or less evident. One is related to interpreting $T^{\prime}$ in $T$ in such a way that elements in $T^{\prime}$ are coded by pairs (triples, etc.) of elements in $T$. Another generalization is related to different strata of formulas : $\forall \ldots \forall \exists . . \exists$ for example.

Section 2 provides a foundation for the method based on the theory of recursive functions. Section 3 is related to a natural question how to start the interpreting process, where to take the first theory with undecidable modest stratum of sentences. As in [2] we begin with Buchi's description of Turing machines by modest first order sentences (see [1]). It produces a strong undecidability result in a natural, readable and elegant way.

In [2] we dealt with recursive inseparability of the set of $\forall p \exists \ldots \exists$ sentences false in a theory $T$ and the set of $\forall^{p} \exists$... $\exists$ sentences satisfiable in finite models of $T$. According to Section 4 the stronger property, effective inseparability, is the right one. In particular recursive inseparability can be replaced by effective inseparability in all results of [2]. That improvement is of course in the spirit of $[7,8,6]$. (An idea of [4] makes the implementation easier. See the definition of p-reducibility in Section 1 , resembling the definition of semi-conservative reduction in [4] and Theorem 1 in Section 4.)

A simple note (see Theorem 3 in Section 4) strengthens the results of [2] in another direction. Let $T$ be a first order theory axiomatizable by a finite number of $\forall^{p} \exists \ldots \exists$ sentences. If the set of $\forall^{p} \exists \ldots \exists$ sentences false in $T$ and the set of $\forall^{P} \exists \ldots \exists$ sentences satisfiable in finite models of $T$ are effectively inseparable then the set of logically false $\forall^{p} \exists \ldots \exists$ sentences in the language of $T$ and the set of $\forall^{p} \exists \ldots \exists$ sentences satisfiable in the finite models of $T$ are effectively inseparable.

According to [9] the set of satisfiable $\forall^{3} \exists \ldots \exists$ sentence in the language of one binary predicate is undecidable. Recursive inseparability of the sets of logically false and finitely satisfiable $\forall^{3} \exists \ldots \exists$ sentences in that language was proved in [2]. (Some errors in that proof were corrected by Lewis [5]. The same result was proved independently by V.F. Kostyrko, unpublished.) That result (in the effective inseparability form) is proved once more in Section 5 and is used for subsequent reductions. It connects this paper with the classical decision problem for fragments of the predicate logic given by a type of prefixes and numbers of unary, binary, etc. predicate symbols. 
Our notation and terminology are more or less standard. We write $\sim \varphi$ for the negation of a formula $\varphi$ and agree that a formula shall be of the form $\alpha \rightarrow \beta$ or $\alpha \leftrightarrow \beta$ rather than $\alpha \wedge \beta$ or $\alpha \vee \beta$ whenever there is a choice. Theorem 1.5 means Theorem 5 of Section 1 similarly Definition 1.6 means Definition 6 of Section 1 . An earlier version of a part of this paper was included into a survey on the classical decision problem withdrawn from the press in USSR when the author left that country. My thanks to Dr. Harry R. Lewis for translating that version into English.

\section{1. p-Reducibility}

All (formal) languages and theories in this paper are first order. Each language is identified with the set of its predicate and function symbols. Individual constants are treated as 0 -ary function symbols. The language of a theory $T$ is denoted by $L(T)$. If the sign of equality belongs to $L(T)$ it is interpreted as the identity in each model of $T$. Each theory is identified with the set of its theorems.

Let $P C$ be an ordinary version of the first order predicate calculus with equality. Each language in this paper is supposed to be a sublanguage and a decidable subset of $L(P C)$.

A sentence $\varphi$ in the language $L(T)$ of a theory $T$ will be called satisfiable (respectively finitely satisfiable) in $T$ if it holds in some model (respectively in some finite model) of $T$. If $\varphi$ is not satisfiable in $T$ it will be called unsatisfiable in $T$ or false in $T$.

An $\forall^{p} \exists \ldots \exists$ formula is a formula $\forall x_{1} \ldots x_{q} \exists y_{1} \ldots y_{n} \psi$ where $q \leqq p$ and $\psi$ is quantifierfree.

Definition 1. A theory $T$ is p-reducible to a theory $T$ if there is an algorithm associating an $\forall^{p} \exists$... $\exists$ sentence $\varphi^{*}$ in $L(T)$ with each $\forall^{p} \exists . . \exists$ sentence $\varphi$ in $L\left(T^{\prime}\right)$ in such a way that

(i) If $\varphi$ is false in $T^{\prime}$ then $\varphi^{*}$ is false in $T$, and

(ii) If $\varphi$ is finitely satisfiable in $T^{\prime}$ then $\varphi^{*}$ is finitely satisfiable in $T$.

Note. For each $i=1,2$ let $T_{i}$ be a theory and $F_{i}$ (respectively $U_{i}$ ) be the set of $\forall^{p} \exists \ldots \exists$ sentences in $L\left(T_{i}\right)$ finitely satisfiable in $T_{i}$ (respectively unsatisfiable in $T_{i}$ ). If $T_{1}$ is p-reducible to $T_{2}$ and there is no decidable set of sentences in $L\left(T_{1}\right)$ separating $F_{1}$ and $U_{1}$ then there is no decidable set of sentences in $L\left(T_{2}\right)$ separating $F_{2}$ and $U_{2}$. For, if $f p$-reduces $T_{1}$ to $T_{2}$ and $Y$ separates $F_{2}, U_{2}$ then $f^{-1} Y$ separates $F_{1}, U_{1}$. If $p=3$ and $T_{1}$ is the theory of one binary predicate then there is not decidable set separating $F_{1}$ and $U_{1}$, that is proved below. These facts give some idea why $p$-reducibility is worth studying.

Lemma 1. The conjunction of $\forall^{p} \exists \ldots \exists$ formulas $\alpha_{1}, \ldots, \alpha_{n}$ is logically equivalent to a single $\forall^{p} \exists \ldots \exists$ formula $\alpha$. Moreover $\alpha$ is computable from $\alpha_{1}, \ldots, \alpha_{m}$ and every free variable of $\alpha$ is among the free variables of $\alpha_{1}, \ldots, \alpha_{n}$. 
Proof is clear.

Definition 2. A theory $T^{\prime}$ is a p-extension of a theory $T$ if $L\left(T^{\prime}\right)=L(T)$ and $T^{\prime}$ extends $T$ by means of a finite number of axioms $\alpha_{1}, \ldots, \alpha_{n}$ such that the universal closure of each $\alpha_{i}$ is equivalent in $T$ to an $\forall^{p} \exists . . \exists$ sentence.

Lemma 2. Each p-extension of a theory $T$ is p-reducible to $T$.

Proof. Let $T^{\prime}$ be a p-extension of $T . T^{\prime}$ is axiomatizable in $T$ by means of a finite number of $\forall^{p} \exists \ldots \exists$ sentences $\beta_{1}, \ldots, \beta_{n^{*}}$. For each $\forall^{p} \exists \ldots \exists$ sentence $\varphi$ in $L(T)$, compute an $\forall^{p} \exists \ldots \exists$ sentence $\varphi^{*}$ logically equivalent to $\beta_{1} \wedge \ldots \wedge \beta_{n} \wedge \varphi$. That gives the desired reduction.

Formulas with exactly $m$ free variables will be called $m$-ary. If both a formula $\varphi$ and its negation $\sim \varphi$ are equivalent to existential formulas in $T$ then $\varphi$ will be called a neutral formula of $T$. The set of neutral formulas of $T$ is closed under the Boolean operations.

Lemma 3. Let $T$ be a theory, $\pi$ be an m-ary neutral formula of $T$, and $T^{\prime}$ be the theory obtained from $T$ by introducing a new m-ary predicate $P$ by means of $\pi$. If $m \leqq p$ then $T^{\prime}$ is $p$-reducible to $T$.

Proof. If $\varphi$ is an $\forall^{p} \exists$... $\exists$ sentence in $L\left(T^{\prime}\right)$ let $\varphi^{\prime}$ be the result of replacing $P$ by $\pi$ in $\varphi$. Compute an $\forall^{p} \exists \ldots \exists$ sentence $\varphi^{*}$ equivalent to $\varphi^{\prime}$ in $T$. That gives the desired reduction.

Recall that elements $a, b$ of a structure $M$ are called indistinguishable if there is no formula $\varphi$ in the language of $M$ with parameters from $M$ such that $\varphi(a)$ holds but $\varphi(b)$ fails in $M$. We'll say that a binary formula $\varepsilon$ in the language of a theory $T$ expresses indistinguishability in $T$ if for every model $M$ of $T$ and elements $a, b$ of $M$, $a, b$ are indistinguishable in $M$ iff $\varepsilon(a, b)$ holds in $M$.

Lemma 4. Let $T$ be a theory without equality, $\varepsilon$ be a neutral formula of $T$ expressing indistinguishability in $T$, and $T$ be the theory obtained from $T$ by introducing equality by means of $\varepsilon$. If $p \geqq 2$ then $T^{\prime}$ is p-reducible to $T$.

Proof. If $\varphi$ is an $\forall^{p} \exists$... $\exists$ sentence in $L\left(T^{\prime}\right)$ let $\varphi^{\prime}$ be the result of replacing equality by $\varepsilon$ in $\varphi$. Compute an $\forall^{p} \exists \ldots \exists$ sentence $\varphi^{*}$ equivalent in $T$ to $\varphi^{\prime}$. That gives the desired reduction.

Lemma 5. Let $T$ be a theory without equality and $\varepsilon$ be a binary neutral formula of $T$. Let $T^{\prime}$ be the theory obtained from $T$ by introducing equality by means of $\varepsilon$. Suppose 
that $L(T)$ is finite, $p \geqq 3$ and $p$ is bigger than the arity of any predicate or function symbol of $T$. Then $T$ is p-reducible to $T$.

Proof. Write a ternary neutral formula $\alpha$ of $T$ whose universal closure expresses that $\varepsilon$ is an equivalence relation. For each $m$-ary predicate symbol $A$ of $T$ write an $(m+1)$-ary neutral formula $\beta_{A}$ of $T$ whose universal closure expresses that $A$ doesn't distinguish $\varepsilon$-equivalent elements. If $m=2$ then $\beta_{A}$ is

$$
\varepsilon\left(x_{1}, x_{2}\right) \rightarrow\left(A x_{1} x_{3} \rightarrow A x_{2} x_{3}\right) \wedge\left(A x_{3} x_{1} \rightarrow A x_{3} x_{2}\right) .
$$

For each $m$-ary function symbol $f$ of $T$ write an $(m+1)$-ary neutral formula $\gamma_{f}$ of $T$ whose universal closure expresses that $f x_{1} \ldots x_{m}, f y_{1} \ldots y_{m}$ are $\varepsilon$-equivalent if $x_{i}, y_{i}$ are $\varepsilon$-equivalent for every $i$. If $m=2$ the $\gamma_{f}$ is

$$
\varepsilon\left(x_{1}, x_{2}\right) \rightarrow \varepsilon\left(f x_{1} x_{3}, f x_{2} x_{3}\right) \wedge \varepsilon\left(f x_{3} x_{1}, f x_{3} x_{2}\right) .
$$

Let $T^{\prime \prime}$ be the extension of $T$ by means of the axioms $\alpha, \beta_{A}, \gamma_{S}$. By Lemma $2 T^{\prime \prime}$ is p-reducible to $T$. By Lemma $4 T^{\prime}$ is $p$-reducible to $T^{\prime \prime}$.

A structure $M$ will be called economical if indistinguishable elements of $M$ are identical. Identifying indistinguishable elements of an arbitrary structure $M$ gives an economical structure which will be called the economical version of $M$.

Definition 3. Let $L$ be a language without function symbols, and $\delta$ be a unary formula in $L$. For each model $M$ for $L$ satisfying $\exists x \delta(x)$ the restriction $M \mid \delta$ of $M$ by $\delta$ is the submodel of $M$ formed by $\{a: \delta(a)$ holds in $M\}$. For each theory $T$ in $L$ the restriction of $T$ by $\delta$ is the theory $T h\{M \mid \delta: M$ is a model of $T$ satisfying $\exists x \delta(x)\}$. The restriction of $T$ by $\delta$ is accurate if for each model $M$ of $T \cup\{\exists x \delta(x)\}$ with finite economical version of $M \mid \delta$ there is a finite model $M^{\prime}$ of $T \cup\{E x \delta(x)\}$ such that the economical versions of $M \mid \delta$ and $M^{\prime} \mid \delta$ are isomorphic.

Lemma 6. Let $T^{\prime}$ be the restriction of a theory $T$ by a formula $\delta$. Suppose it is an accurate restriction.

(i) Each finite model of $T^{\prime}$ is elementarily equivalent to the restriction of some finite model of $T$ by $\delta$.

(ii) If $\delta$ is neutral then $T^{\prime}$ is p-reducible to $T$ for any $p$.

Proof. For each formula $\alpha$ let $\alpha \mid \delta$ be the result of restricting quantifiers by $\delta$ in $\alpha$.

(i) If $N$ is a model of $T^{\prime}$ then

$$
T \cup\{\exists x \delta(x)\} \cup\{\alpha \mid \delta: \alpha \in \operatorname{Th}(N)\}
$$

is consistent and has a model $M$. Clearly $M \mid \delta$ is elementarily equivalent to $N$. If $N$ is finite then the economical version of $M \mid \delta$ is finite and, thanks to accuracy of the restriction, there is a finite model $M^{\prime}$ of $T$ such that the economical versions of $M \mid \delta$ and $M^{\prime} \mid \delta$ are isomorphic. $M^{\prime}$ is the desired model. 
(ii) For each $\forall^{p} \exists . . \exists$ sentence $\varphi$ in $L(T)$ compute an $\forall^{p} \exists \ldots \exists$ sentence $\varphi^{*}$ equivalent to $\exists x \delta(x) \wedge(\varphi \mid \delta)$ in $T$.

Definition 5. Suppose $L \subseteq L(T)$ i.e. $L$ is a sublanguage of the language of a theory $T$. For each model $M$ for $L(T)$ let $M \mid L$ be the reduction of $M$ to $L$. The theory

$$
\text { Th }\{M \mid L: M \text { is a model of } T\}
$$

is the reduction of $T$ to $L$. The reduction of $T$ to $L$ is accurate if for each model $M$ of $T$ with finite economical version of $M \mid L$ there is a finite model $M^{\prime}$ of $T$ such that the economical versions of $M \mid L$ and $M^{\prime} \mid L$ are isomorphic.

Note that the restriction of $T$ to $L$ is the intersection of $T$ and the set of formulas in $L$.

Lemma 7. Let $T$ be the reduction of $T$ to a sublanguage $L$ of $L(T)$. Suppose it is an accurate reduction.

(i) Each finite model of $T^{\prime}$ is elementarily equivalent to the reduction of some finite model of $T$.

(ii) $T^{\prime}$ is p-reducible to $T$ for any $p$.

Proof. (i) If $N$ is a model of $T^{\prime}$ then $T \cup T h(N)$ is consistent and has a model $M$. Clearly $M \mid L$ is elementarily equivalent to $N$. If $N$ is finite then the economical version of $M \mid L$ is finite and, thanks to accuracy of the reduction, there is a finite model $M^{\prime}$ of $T$ such that the economical versions of $M \mid L$ and $M^{\prime} \mid L$ are isomorphic.

$M^{\prime}$ is the desired model.

(ii) Take $\varphi^{*}$ be equal to $\varphi$.

Definition 6. Let $T^{\prime}$ be a theory in a language $L^{\prime}$ without equality. $T^{\prime}$ is embedded into $T$ if

(1) $L^{\prime} \leqq L(T)$,

(2) the reduction of $T$ to $L^{\prime}$ extends $T^{\prime}$, and

(3) for each finite economical model $N$ of $T^{\prime}$ there is a finite model $M$ of $T$ such that the economical version of $M \mid L^{\prime}$ is isomorphic to $N$.

Lemma 8. If a theory $T^{\prime}$ without equality is embedded into a theory $T$ then $T^{\prime}$ is p-reducible to $T$ for any $p$.

Proof. Take $\varphi^{*}$ be equal to $\varphi$.

Definition 7. Let $L^{\prime}$ be a language without equality and $T^{\prime}$ be a theory in the language $L^{\prime} \cup\{=\}$. Let $\varepsilon$ be a binary neutral formula of a theory $T$. $T^{\prime}$ is embedded into $T$ modulo $\varepsilon$ if the following conditions are satisfied.

(1) $L^{\prime} \leqq L(T)$.

(2) For each model $M$ of $T, \varepsilon$ gives an equivalence relation on $|M|$ and $\varepsilon$-equivalent elements are indistinguishable in $M \mid L^{\prime}$ and the quotient structure $\left(M \mid L^{\prime}\right) / \varepsilon$ is a model of $T^{\prime}$. 
(3) For each finite model $N$ of $T^{\prime}$ there is a finite model $M$ of $T$ such that $\left(M \mid L^{\prime}\right) / \varepsilon$ is elementarily equivalent to $N$.

Lemma 9. If a theory $T$ with equality is embedded into a theory $T$ modulo a formula $\varepsilon$ of $T$ then $T^{\prime}$ is p-reducible to $T$ for any $p$.

Proof. For each $\forall^{p} \exists \ldots \exists$ sentence $\varphi$ in $L\left(T^{\prime}\right)$ compute an $\forall^{p} \exists . . \exists$ sentence $\varphi^{*}$ equivalent in $T$ to the result of replacing equality by $\varepsilon$ in $\varphi$. It gives the desired reduction.

\section{Effective Inseparability}

Let $W_{0}, W_{1}, \ldots$ be a canonical numeration of recursively enumerable sets of natural numbers, and $A, B, C, D$ be sets of natural numbers. Recall that $A, B$ are called effectively inseparable if there exists a recursive binary function $F$ (under which $A$, $B$ are said to be effectively inseparable) such that for every disjoint $W_{m}$ and $W_{n}$, if $A \subseteq W_{n}$ and $B \leqq W_{n}$ then $F(m, n) \notin W_{m} \cup W_{n}$. We cite that definition as well as most statements of this section from [8].

Corollary 1. If $A, B$ are effectively inseparable they are recursively inseparable i.e., there is no recursive $C$ including one of them and disjoint from the other.

Corollary 2. Let $C, D$ be disjoint and $A \subseteq C, B \subseteq D$. If $A, B$ are effectively inseparable then so are $C, D$.

Theorem 3. There exists a pair of recursively enumerable effectively inseparable sets.

Proof. See Section 1 in Chapter V of [8].

Theorem 4. Let $A, B$ be recursively enumerable and effectively inseparable. For every disjoint and recursively enumerable $C, D$ there exists a recursive function $f$ such that $C=f^{-1}(A)$ and $D=f^{-1}(B)$.

Proof. See Section 17 in Chapter V of [8].

Theorem 5. Let $f(A) \subseteq C$ and $f(B) \subseteq D$ where $A, B$ are recursively enumerable and effectively inseparable, $f$ is a recursive function and $C, D$ are disjoint. Then $C, D$ are effectively inseparable. Moreover there exists a recursive binary function $F$ such that $C, D$ are effectively inseparable under $F$ and the range of $f$ includes the range of F.

Proof. See Proposition 4 and its proof in Chapter V of [8].

The next theorem is essentially Theorem 1 in [4]. 
Theorem 6. Let $A, B, C, D, f$ be as in Theorem 5. There exists a recursive function $g$ such that $A=g^{-1}(C), B=g^{-1}(D)$ and the range of $f$ includes the range of $g$.

Proof. Without loss of generality $C=f(A)$ and $D=f(B)$. By Theorem 5 there is an $F$ such that $C, D$ are effectively inseparable under $F$ and $\operatorname{Rng}(F) \leqq \operatorname{Rng}(f)$. By Theorem $12 \mathrm{~b}$ and its proof in Chapter $\mathrm{V}$ of [8] there is a recursive binary function $G$ such that $\operatorname{Rng}(G) \subseteq \operatorname{Rng}(F)$ and $C, D$ are doubly creative under $G$. (The definition of double creativity may be found in [8] too.) By Theorem 15(1) and its proof in Chapter V of [8] there exists a recursive function $g$ such that $A=g^{-1}(C)$, $B=g^{-1}(D)$ and $\operatorname{Rng}(g) \subseteq \operatorname{Rng}(G)$.

\section{Buchi's Reduction}

Fix a certain Gödel numbering of Turing machines. Let $\mathrm{Ha}$ (respectively $\mathrm{Ci}$ ) be the set of numbers of Turing machines $M$ such that $M$ halts (respectively enters a circle) starting on a blank tape in the initial state.

Lemma 1. $\mathrm{Ha}$ and $\mathrm{Ci}$ are effectively inseparable.

Proof. Let $A, B$ be recursively enumerable effectively inseparable sets of natural numbers. Define $f n=0$ if $n \in A$, and $f n=1$ if $n \in B$. $f$ is a recursive partial function, hence there is a Turing machine $M^{\prime}$ computing $f$. A simple adjunct turns $M^{\prime}$ into a Turing machine $M^{*}$ which halts if $n \in A$, and enters a circle if $n \in B$. For each $n$ let $M_{n}^{\prime}$ be a Turing machine computing the constant function $n$, and $M_{n}$ be the concatenation of $M_{n}^{\prime}$ and $M^{*}$. Let $g(n)$ be the number of $M_{n}$. Then $g(A) \subseteq H a$ and $g(B) \subseteq C i$. Now use Theorem 2.5.

With each language in this paper we associate a certain Gödel numbering of all formulas in that language. Formulas are identified with their numbers. Below $P, Q$, $P_{i}, Q_{i}$ are binary predicate symbols and $R, R_{j}$ are unary ones.

In [1] each Turing machine $M$ is described by a sentence

$$
\alpha_{M}=\left(\exists x R_{1} x\right) \wedge \forall x \exists y \forall z \alpha_{M}^{\prime}
$$

in the language $\left\{P_{1}, P_{2}, P_{3}, R_{1}, R_{2}, \ldots\right\}$ in such a way that $\alpha_{M}$ is logically false if $M \in H a$, and $\alpha_{M}$ is finitely satisfiable (i.e., satisfiable in a finite set) if $M \in C i$. Actually $\alpha_{M}$ is computable from $M$.

Corollary 2. The sets of logically false and finitely satisfiable $\forall^{3} \exists \ldots \exists$ sentences in the language $\left\{P_{1}, P_{2}, P_{3}, P_{4}, R_{1}, R_{2}, \ldots\right\}$ are effectively inseparable.

Proof. For each Turing machine $M$ let

$$
\beta_{M}=\left(\exists x R_{1} x\right) \wedge\left(\forall x \exists y P_{4} x y\right) \wedge \forall x y z\left(P_{4} x y \rightarrow \alpha_{M}^{\prime}\right) .
$$


Every model of $\beta_{M}$ is a model of $\alpha_{M}$. An appropriate definition of $P_{4}$ turns each model of $\alpha_{M}$ into a model of $\beta_{M}$. If $M \in H a$ then $\alpha_{M}$ is logically false hence $\beta_{M}$ is logically false. If $M \in C i$ then $\alpha_{M}$ is finitely satisfiable hence $\beta_{M}$ is finitely satisfiable. Now use Lemma 1 and Theorem 2.5.

\section{4. $p$-Inseparability}

Fix a natural number $p \geqq 3$. For each theory $T$ let $F S(T)$ [respectively $U S(T)$ ] be the set of those $\forall^{p} \exists \ldots \exists$ sentences in the language $L(T)$ of $T$ which are finitely satisfiable (respectively unsatisfiable) in $T$. Let $L F(T)$ be the set of logically false sentences in $L(T)$.

Definition 1. A theory $T$ is p-inseparable if $F S(T)$ and $U S(T)$ are effectively inseparable.

Corollary 0. PC (a version of the predicate calculus) is p-inseparable.

Proof. See Corollary 3.2 .

Theorem 1. Suppose that a p-inseparable theory $T^{\prime \prime}$ is p-reducible to a theory $T$ and $F S\left(T^{\prime}\right), U S\left(T^{\prime}\right)$ are recursively enumerable. Then $T$ is $p$-inseparable and there is a recursive function $g$ associating an $\forall \forall^{p} \exists . . \exists$ sentence $g(\varphi)$ in $L(T)$ with each $\forall p \exists \ldots \exists$ sentence $\varphi$ in $L\left(T^{\prime}\right)$ in such a way that (i) $\varphi \in F S\left(T^{\prime}\right)$ iff $g(\varphi) \in F S(T)$, and (ii) $\varphi \in U S\left(T^{\prime}\right)$ iff $g(\varphi) \in U S(T)$.

Proof. Let an algorithm $f p$-reduce $T^{\prime}$ to $T$. Algorithms are identified here with recursive partial functions. Without loss of generality $f$ is total and the range of $f$ consists of $\forall p \exists \ldots \exists$ sentences in $L(T)$. Now use Theorems 2.5 and 2.6 .

Theorem 2. Suppose $F S(T)$ and $U S(T)$ are recursively enumerable. If $T$ is p-inseparable then $P C$ is p-reducible to $T$.

Proof. By Theorem 2.3 there is a recursive function $f$ such that $F S(P C)=f^{-1}(F S(T))$ and $U S(P C)=f^{-1}(U S(T))$. There is an $\forall^{p} \exists \ldots \exists$ sentence $\psi$ in $L(T)$ which is satisfiable in $T$ but isn't finitely satisfiable in $T$, otherwise $F S(T)$ and $U S(T)$ are recursive. Define $g(n)=f(n)$ if $f(n)$ is an $\forall^{p} \exists \ldots \exists$ sentence in $L(T)$, and $g(n)=\psi$ otherwise. $g$ is the defined reduction.

Theorem 3. Suppose that $T$ is axiomatizable by a finite number of $\forall p \exists \ldots \exists$ sentences. If $T$ is p-inseparable then $F S(T)$ and $L F(T)$ are effectively inseparable.

Proof. By Theorem 2 there is a recursive function $f p$-reducing $P C$ to $T$. Let $\alpha_{1}, \ldots, \alpha_{m}$ be $\forall^{p} \exists \ldots \exists$ sentences axiomatizing $T$. For each $\forall^{p} \exists \ldots \exists$ sentence $\varphi$ in $L(P C)$ 
compute an $\forall^{p} \exists . . \exists$ sentence $g(\varphi)$ logically equivalent to $\alpha_{1} \wedge \ldots \wedge \alpha_{m} \wedge f(\varphi)$. If $\varphi$ is finitely satisfiable then $g(\varphi) \in F S(T)$. If $\varphi$ is logically false then $g(\varphi) \in L F(T)$. Now use Theorem 2.5.

\section{One Binary Predicate}

Recall that $P, Q, P_{1}, \ldots$ are used for binary predicate symbols, and $R, R_{1}, \ldots$ are used for unary predicate symbols. If every theorem of a theory $T$ is logically true we say $T$ is a logic.

Theorem 1. The logic of 5 binary predicates plus equality is 3-inseparable.

Proof. For each $m>1$ let $S_{m}$ be the logic in $\left\{P_{1}, P_{2}, P_{3}, P_{4}, R_{1}, \ldots, R_{m}\right\}$. Let $T_{0}$ be the logic in $\left\{P_{1}, P_{2}, P_{3}, P_{4}, Q,=\right\}$. In virtue of Corollary 3.2 and Theorem 4.1 it suffices to prove that $S_{m}$ is 3-reducible to $T$ uniformly in $m$.

Let $\sigma(x, y)$ abbreviate $Q x x \wedge Q y y \wedge Q x y \wedge \sim Q y x, v_{1}(x)$ abbreviate $Q x x \wedge P_{1} x x$, and $v_{i+1}(x)$ abbreviate $\exists y\left(v_{i}(y) \wedge \sigma(y, x)\right)$ for $1 \leqq i<m$. Every $v_{i}$ is logically equivalent to an existential formula. Let $T_{1}$ be the 3-extension of $T_{0}$ by means of the axioms

$$
\begin{gathered}
\exists x v_{m}(x), \\
v_{1}(x) \wedge v_{1}(y) \rightarrow x=y, \\
\sigma(x, y) \wedge \sigma(x, z) \rightarrow y=z .
\end{gathered}
$$

Each $v_{i}$ is neutral in $T_{1}$ for its negation is equivalent to $\exists y\left(v_{i}(y) \wedge x \neq y\right)$ in $T_{1}$.

Let $T_{2}$ be the theory obtained from $T_{1}$ by introducing unary predicates $R_{1}, \ldots, R_{m}$ by means of formulas $\exists y\left(v_{1}(y) \wedge Q y x\right), \ldots, \exists y\left(v_{m}(y) \wedge Q y x\right)$. These formulas are neutral in $T_{1}$ because the negation of $\exists y\left(v_{i}(y) \wedge Q y x\right)$ is equivalent in $T_{1}$ to $\exists y\left(v_{i}(y) \wedge \sim Q y x\right)$.

Let $T_{3}$ be the restriction of $T_{2}$ by the formula $\sim Q \times x$. It is an accurate restriction of course. Now we check that $S_{m}$ is embedded into $T_{3}$. Condition (3) of Definition 1.6 is the only one we need to check. Given a model $N$ of cardinality $n$ build a model $M$ of $T_{2}$ of cardinality $n+m$ such that $(M \mid \sim Q) \mid L\left(S_{m}\right)$ is isomorphic to $N$.

By Section 1 every successor in the sequence $T_{0}, T_{1}, T_{2}, T_{3}, S_{m}$ is 3-reducible to its predecessor, hence $S_{m}$ is 3-reducible to $T_{0}$. Section 1 gives also the actual reduction, which is uniform in $m$.

Theorem 2. The logic of a binary predicate is 3-inseparable.

Proof. Let $T_{0}$ be the logic of a binary predicate $P$ and $T_{1}$ be the 3-extension of $T_{0}$ by means of the axioms

$$
\begin{gathered}
\exists x P x x, \\
P x x \wedge P y y \rightarrow(P x z \rightarrow P y z) \wedge(P z x \rightarrow P z y) .
\end{gathered}
$$

Any economical model of $T_{1}$ contains exactly one $P$-reflexive element. 
Let $T_{2}$ be the theory obtained from $T_{1}$ by introducing unary predicate $R_{0}, R_{1}, R_{2}$, $R_{3}$ by means of the formulas

$$
\sim P x x \wedge \exists y(P y y \wedge \pm P x y \wedge \pm P y x),
$$

which are neutral in $T_{1}$. Let $T_{3}$ be the theory obtained from $T_{2}$ by introducing equality by means of the formula

$$
(P x x \wedge P y y) \vee\left(\sim P x y \wedge \bigvee\left\{R_{i} x \wedge R_{i} y: 0 \leqq i \leqq 3\right\}\right) .
$$

A model $M$ for $L\left(T_{3}\right)$ is a model of $T_{3}$ iff it has exactly one $P$-reflexive element, $P$-irreflexive elements of $M$ are partitioned by $R_{0}, R_{1}, R_{2}, R_{3}$ in four classes and different elements in the same class are two-way connected by $P$. Let $T_{4}$ be the 3-extension of $T_{3}$ by means of the axioms

$$
\begin{gathered}
\exists x R_{0} x, \\
R_{0} x \rightarrow \exists y\left(R_{i} x \wedge P x y\right), \\
R_{0} x \wedge R_{i} y \wedge R_{i} z \wedge P x y \wedge P x z \rightarrow y=z,
\end{gathered}
$$

where $1 \leqq i \leqq 3$. They state that for every $R_{0}$-element $x$ and every $i=1,2,3$ there is exactly one $R_{i}$-element $y$ such that $P x y$ holds.

Let $T_{5}$ be the theory obtained from $T_{4}$ by introducing binary predicates $Q_{1}, \ldots, Q_{5}$ by means of the formulas

$$
R_{0} x \wedge R_{0} y \wedge \exists x^{\prime} y^{\prime}\left(R_{i} x^{\prime} \wedge R_{j} y^{\prime} \wedge P x x^{\prime} \wedge P y y^{\prime} \wedge P x^{\prime} y^{\prime}\right),
$$

where $(i, j)$ is $(1,2),(2,1),(1,3),(3,1)$ or $(2,3)$ respectively. All 5 formulas are neutral in $T_{4}$.

Let $T_{6}$ be the restriction of $T_{5}$ by $R_{0}$. It is an accurate restriction of course. Let $T_{7}$ be the logic in $\left\{Q_{1}, \ldots, Q_{5},=\right\} . T_{7}$ is embedded into $T_{6}$ modulo equality. [Given a model $N$ of $T_{7}$ of cardinality $n$ build a model $M$ of $T_{5}$ of cardinality $4 n+1$ such that $\left(M \mid R_{0}\right) \mid L\left(T_{7}\right)$ is isomorphic to $N$.] By Section 1 every successor in the sequence $T_{0}, \ldots, T_{7}$ is 3 -reducible to its predecessor hence $T_{7}$ is 3-reducible to $T_{0}$. By Theorem $1 T_{7}$ is 3 -inseparable hence $T_{0}$ is 3 -inseparable.

\section{One Symmetric Predicate}

Lemma 1. Let $T$ be the theory in the language $\left\{P, R_{0}, R_{1}, R_{2}\right\}$ whose axioms state

(i) $P$ is irreflexive and symmetric, and

(ii) $R_{0}, R_{1}, R_{2}$ partition the universe i.e. each element satisfy exactly one of these 3 unary predicates.

Then $T$ is 3-inseparable.

Proof. Let $T_{1}$ be the theory obtained from $T$ by introducing equality by means of the formula

$$
\sim P x y \wedge \bigvee\left\{R_{i} x \wedge R_{i} y: 0 \leqq i \leqq 2\right\}
$$


In each model of $T_{1}$ different elements satisfying the same $R_{i}$ are connected by $P$. Let $T_{2}$ be the extension of $T_{1}$ by means of the axioms

$$
\begin{gathered}
R_{0} x \rightarrow \exists y\left(R_{i} y \wedge P x y\right), \\
R_{0} x \wedge R_{i} y \wedge R_{i} z \wedge P x y \wedge P x z \rightarrow y=z,
\end{gathered}
$$

where $i=1,2$. They state that each $R_{0}$-element is linked by $P$ with exactly one $R_{1}$-element and exactly one $R_{2}$-element.

Let $T_{3}$ be obtained from $T_{2}$ by introducing a predicate $Q$ by the formula

$$
R_{0} x \wedge R_{0} y \wedge \exists x^{\prime} y^{\prime}\left(R_{1} x^{\prime} \wedge P x x^{\prime} \wedge R_{2} y^{\prime} \wedge P y y^{\prime} \wedge P x^{\prime} y^{\prime}\right),
$$

which is neutral in $T_{2}$.

Let $T_{4}$ be the (accurate) restriction of $T_{3}$ by $R_{0}$.

Let $T_{5}$ be the logic of a binary predicate $Q$. We prove that $T_{5}$ is embedded into $T_{4}$ with respect to Definition 1.6. The condition (3) of that definition is the only one we need to check. Let $N$ be a finite economical model of $T_{5}$. Build a model $M$ for $L\left(T_{3}\right)$ as follows:

$|M|=|N| \times\{0,1,2\}$, we write $a_{i}$ for $\langle a, i\rangle$.

$R_{i} a_{j}$ is true iff $i=j$.

$P a_{i} b_{i}$ is true iff $a \neq b . P a_{0} b_{1}, P b_{1} a_{0}, P a_{0} b_{2}, P b_{2} a_{0}$ are true if $a=b$, and false otherwise. $P a_{1} b_{2}, P b_{2} a_{1}$ are true if $Q a b$ holds in $N$, and false otherwise.

$Q a_{i} b_{j}$ is true if $i=0, j=0$ and $Q a b$ holds in $N$.

Clearly $M$ is a model of $T_{3}$. Hence $M \mid R_{0}$ is a model of $T_{4}$. But it is finite and $\left(M \mid R_{0}\right) \mid\{Q\}$ is isomorphic to $N$.

By Section $1 T_{5}$ is 3-reducible to $T$. By Section $5 T_{5}$ is 3-inseparable. Hence $T$ is 3-inseparable. Q.E.D.

Theorem 2. Let $T$ be the theory in the language $\{P, R\}$ whose only axiom states that $P$ is irreflexive and symmetric. Then $T$ is 3-inseparable.

Proof. Let $T_{1}$ be the extension of $T$ by the axioms

$$
\begin{gathered}
\exists x y(R x \wedge R y \wedge \sim P x y), \\
(R x \wedge R y \wedge R z) \rightarrow(P x y \vee P y z \vee P z x), \\
(R x \wedge R y \wedge P x y) \rightarrow(P x z \rightarrow P y z) .
\end{gathered}
$$

In each economical model of $T_{1}$ there are exactly two elements satisfying $R$ and they are not connected by $P$.

Let $T_{2}$ be the theory obtained from $T_{1}$ by introducing unary predicates $R_{i}(i=1,2,3)$ by means of the formulas

$$
\sim R x \wedge \exists y z\left(R y \wedge R z \wedge \sim P y z \wedge \alpha_{i}\right)
$$


where

$$
\begin{gathered}
\alpha_{0}=\sim P x y \wedge \sim P x z, \\
\alpha_{1}=\sim(P x y \leftrightarrow P x z), \\
\alpha_{2}=P x y \wedge P x z .
\end{gathered}
$$

It is easy to see that the formulas defining $R_{0}, R_{1}, R_{2}$ are neutral in $T_{1}$.

Let $T_{3}$ be the restriction of $T_{2}$ by $\sim R x$. It is an accurate restriction.

Let $T_{4}$ be theory of Lemma 1 . It is easy to see that $T_{4}$ is embedded into $T_{3}$. In particular each model $N$ of $T_{4}$ of cardinality $n$ can be augmented to a model $M$ of $T_{2}$ of cardinality $n+2$ in such a way that $(M \mid \sim R) \mid L\left(T_{4}\right)$ is isomorphic to $N$.

By Section $1 T_{4}$ is 3-reducible to $T$. By Lemma $1 T_{4}$ is 3-inseparable. Hence $T$ is 3-inseparable. Q.E.D.

Theorem 3. The theory of one binary symmetrical predicate is 3-inseparable.

Proof. Replace $R u$ by Puu in the proof of Theorem 2 .

\section{One Irreflexive Predicate}

Theorem 1. The theory of one irreflexive binary predicate is 3-inseparable.

Proof. Let $T_{0}$ be the theory in $\{P\}$ whose only axiom states that $P$ is irreflexive. Let $\alpha(x, y)$ abbreviate $P x y \wedge \sim P y x$ and $T_{1}$ be the extension of $T_{0}$ by means of the axioms

$$
\begin{aligned}
& \exists y(\alpha(x, y) \vee \alpha(y, x)), \\
& \sim(\alpha(x, y) \wedge \alpha(y, z)) .
\end{aligned}
$$

Let $T_{2}$ be the theory obtained from $T_{1}$ by introducing a unary predicate $S$ by the formula $\exists y \alpha(y, x)$. The negation of that formula is equivalent to $\exists y \alpha(x, y)$ in $T_{1}$ hence it is neutral in $T_{1}$. For each model $M$ of $T_{2}$ the predicate $P$ is irreflexive and symmetric in both submodels $M \mid S$ and $M \mid \sim S$.

Let $T_{3}$ be the extension of $T_{2}$ by the axioms

$$
\begin{gathered}
S x \wedge S y \wedge \sim P x y \rightarrow(P x z \rightarrow P y z) \wedge(P z x \rightarrow P z y), \\
S x \wedge S y \wedge S z \rightarrow \sim P x y \vee \sim P y z \vee \sim P z x, \\
\exists x y(S x \wedge S y \wedge P x y) .
\end{gathered}
$$

In each economical model of $T_{3}$ there are exactly two elements satisfying $S$ and they are connected by $P$.

Let $T_{4}$ be the theory obtained from $T_{3}$ by introducing a unary predicate $R$ by means of the formula

$$
\exists y z(S y \wedge S z \wedge P y z \wedge \alpha(x, y) \wedge \alpha(x, z))
$$


which is neutral in $T_{3}$ for its negation is equivalent in $T_{3}$ to

$$
S x \vee \exists y(S y \wedge \sim \alpha(x, y)) .
$$

Let $T_{5}$ be the restriction of $T_{4}$, by $\sim S$. It is an accurate restriction.

Let $T_{6}$ be the theory of Theorem 6.2. It is easy to see that $T_{6}$ is embedded into $T_{5}$. In particular each model $N$ of $T_{6}$ of cardinality $n$ can be augmented to a model $M$ of $T_{4}$ of cardinality $n+2$ in such a way that $(M \mid \sim S) \mid L\left(T_{6}\right)$ is isomorphic to $N$. By Section $1 T_{6}$ is 3-reducible to $T_{0}$. By Section $6 T_{6}$ is 3-inseparable. Hence $T_{0}$ is 3-inseparable.

\section{One Irreflexive Symmetrical Predicate and Equality}

Theorem 1. The theory of equality and one irreflexive symmetrical predicate is 5-inseparable.

Proof. Let $T_{0}$ be the theory of equality and an irreflexive symmetrical predicate $P$. Let

$$
\triangle(x, y, z), \delta(x, y), \varepsilon(x, y)
$$

abbreviate the formulas

$$
P x y \wedge P y z \wedge P z x, \exists z \triangle(x, y, z), \exists z(\delta(x, z) \wedge \delta(y, z))
$$

respectively. We say that $x, y, z$ form a triangle if $\Delta(x, y, z)$ holds, and $x$ is a vertex if there are $y, z$ such that $x, y, z$ form a triangle, and $x$ is a point if it is not a vertex. Let $T_{1}$ be the 5-extension of $T_{0}$ by means of the axioms

$$
\begin{gathered}
\Delta(x, y, z) \wedge \triangle(x, u, w) \rightarrow y=u \vee y=w, \\
P x y \rightarrow \exists u w(\triangle(x, u, w) \vee \triangle(y, u, w)) .
\end{gathered}
$$

The first axiom states that different triangles can't have a common vertex. Hence $\varepsilon$ is an equivalence relation of vertices. Every triangle is an equivalence class of $\varepsilon$. The second axiom states that two points can't be linked by $P$. Hence $\sim \delta(x, y)$ is equivalent in $T_{1}$ to an existential formula stating that either $x, y$ are not linked by $P$ or there is a triangle containing exactly one of them. Hence $\delta$ is neutral in $T_{1}$.

Let $T_{2}$ be the extension of $T_{1}$ by means of the axioms

$$
\begin{gathered}
\delta\left(x, x^{\prime}\right) \wedge \delta\left(y, y^{\prime}\right) \wedge \sim \delta(x, y) \rightarrow \sim P x y, \\
\exists y P x y .
\end{gathered}
$$

The first axiom states that vertices of different triangles can't be linked by $P$. Let $T_{3}$ be the theory obtained from $T_{2}$ by introducing a unary predicate $R$ by means of the formula $\exists y \delta(x, y)$, stating that $x$ is a vertex. That formula is neutral in $T_{2}$ for its negation is equivalent in $T_{2}$ to an existential formula

$$
\exists y u z(\triangle(y, z, u) \wedge P x y \wedge \sim P x z \wedge \sim P x u) \text {. }
$$


Let $T_{4}$ be the theory obtained from $T_{3}$ by introducing a binary predicate $E$ by means of the formula $\varepsilon(x, y)$, which is neutral in $T_{3}$ for $\sim \varepsilon(x, y)$ is equivalent in $T_{3}$ to

$$
R x \wedge R y \rightarrow x+y \wedge \sim \delta(x, y)
$$

Let $\alpha(x, u, y)$ abbreviate

$$
\exists x^{\prime} y^{\prime}\left(E x x^{\prime} \wedge E y y^{\prime} \wedge \sim E x y \wedge P x^{\prime} u \wedge P u y^{\prime}\right)
$$

stating that $x, y$ are vertices, their triangles are different and connected through the point $u$.

Let $T_{5}$ be the extension of $T_{4}$ by means of the axioms

$$
\begin{aligned}
& R x \wedge R y \wedge \sim E x y \rightarrow \exists u w(\alpha(x, u, y) \wedge \alpha(x, w, y) \wedge u \neq w), \\
& \alpha(x, u, y) \wedge \alpha(x, v, y) \wedge \alpha(x, w, y) \rightarrow u=v \vee v=w \vee w=u,
\end{aligned}
$$

stating that two different triangles are connected through exactly two points.

Let $T_{6}$ be the theory obtained from $T_{5}$ by introducing a binary predicate $Q x y$ by means of the formula

$$
\exists u w x^{\prime}\left(\alpha(x, u, y) \wedge \alpha(x, w, y) \wedge u \neq w \wedge E x x^{\prime} \wedge P x^{\prime} u \wedge P x^{\prime} w\right),
$$

stating that $x, y$ are vertices of different triangles and the two points connecting the triangles are linked with the same vertex of the triangle of $x$. That formula is neutral in $T_{5}$ for its negation in equivalent in $T_{5}$ to an existential formula stating that if $x, y$ are vertices of different triangles then there are two points connecting the triangles and linked to different vertices of the triangle of $x$.

Let $T_{7}$ be the restriction of $T_{6}$ by $R$. It is an accurate restriction of course. Let $T_{8}$ be the reduction of $T_{7}$ to the sublanguage $L=\{E, Q\}$ of $L\left(T_{7}\right)$. Again, it's an accurate reduction.

Let $T_{9}$ be the theory of an irreflexive predicate $Q$. We prove that $T_{9}$ is embedded into $T_{8}$. Condition (3) of Definition 1.6 is the only one we need to check.

If $N$ is a finite model of $T_{9}$ build a model $M$ of $T_{6}$ as follows:

$$
\begin{gathered}
|M|=\left\{a_{i}: a \in|N| \text { and } i \in\{1,2,3\}\right\} \cup \\
\{(a, b): a, b \in|N| \text { and } a \neq b\} .
\end{gathered}
$$

$P a_{i} a_{j}$ holds if $i \neq j . P a_{1}(a, b)$ holds always. If $Q a b$ holds in $N$ then $P a_{1}(b, a)$ holds. If $Q a b$ fails in $N$ then $P a_{2}(b, a)$ holds. $P$ fails in all other cases.

$R a_{i}$ holds, $R(a, b)$ fails.

$E\left(a_{i}, a_{j}\right)$ holds, $E$ fails in all other cases.

$Q\left(a_{i}, b_{j}\right)$ holds if $Q a b$ holds in $N, Q$ fails in all other cases.

Let $M^{\prime}=M\left|R, M^{\prime \prime}=M^{\prime}\right|\{E, Q\} . M^{\prime \prime}$ is a finite model of $T_{8}$ and the economical versions of $M^{\prime \prime} \mid\{Q\}$ and $N$ are isomorphic.

By Section $7 T_{9}$ is 3-inseparable. Hence it is 5-inseparable. Use Section 1 to check that theories $T_{8}, \ldots, T_{0}$ are 5-inseparable. 


\section{One Irreflexive Symmetrical Predicate}

Theorem 1. The theory of one irreflexive symmetrical predicate is 5-inseparable.

Proof. Let $T_{0}$ the the theory of an irreflexive symmetrical predicate $P, \triangle(x, y, z)$ abbreviate $P x y \wedge P y z \wedge P z x, \delta(x, y)$ abbreviate $\exists z \Delta(x, y, z)$. Let $T_{1}$ be the extension of $T_{0}$ by means of the axioms

$$
\begin{gathered}
\exists y z \Delta(x, y, z), \\
\Delta\left(x, y, z_{1}\right) \wedge \Delta\left(x, y, z_{2}\right) \wedge \sim P z_{1} z_{2} \rightarrow\left(P u z_{1} \rightarrow P u z_{2}\right) .
\end{gathered}
$$

Let $T_{2}$ be the theory obtained from $T_{1}$ by introducing equality by means of the formula

$$
\sim P x y \wedge \exists z u(\Delta(x, z, u) \wedge \triangle(y, z, u)),
$$

which is neutral in $T_{1}$ for its negation is equivalent in $T_{1}$ to

$$
\exists z \sim(P x z \leftrightarrow P y z) .
$$

Let $T_{3}$ be the extension of $T_{2}$ by means of the axiom

$$
\triangle(x, y, z) \wedge \triangle(x, u, w) \rightarrow y=u \vee y=w .
$$

$T_{3}$ is the theory with equality of models $M$ of $T_{0}$ such that each element of $M$ belongs to exactly one triangle in $M$. Let $\varepsilon(x, y)$ abbreviate $x=y \vee \delta(x, y)$. Then $\varepsilon$ is an equivalence relation in $T_{3} . \delta(x, y)$ is neutral in $T_{3}$ for its negation is equivalent in $T_{3}$ to

$$
P x y \rightarrow \exists u w(\triangle(x, u, w) \wedge y \neq u \wedge y \neq w) .
$$

Hence $\varepsilon$ is neutral in $T_{3}$.

Let $T_{4}$ be the theory obtained from $T_{3}$ by introducing a binary predicate $Q$ by means of the formula

$$
\sim \varepsilon(x, y) \wedge \exists u w(\varepsilon(x, u) \wedge \varepsilon(y, w) \wedge P u w) .
$$

That formula is neutral in $T_{3}$ : its negation states that either $x, y$ belong to the same triangle or no vertex of the triangle of $x$ is connected with any vertex of the triangle of $y$.

Let $T_{5}$ be the theory of equality and an irreflexive symmetrical predicate $Q$. We prove that $T_{5}$ is embedded into $T_{4}$ modulo $\varepsilon$. The condition (3) of Definition 1.7 is the only one we need to check. Let $N$ be a finite economical model of $T_{5}$. Build a model $M$ for $L\left(T_{4}\right)$ as follows.

$$
|M|=|N| \times\{1,2,3\} .
$$

$P(a, i)(a, j)$ holds if $i \neq j$. If $Q a b$ holds in $N$ then $P(a, 1)(b, 2)$ and $P(b, 2)(a, 1)$ hold. $P$ fails in all other cases.

$Q(a, i)(b, j)$ holds iff $Q a b$ holds in $N$. 
It is easy to see that $M$ is a finite model of $T_{4}$. For each $a$ the elements $(a, 1),(a, 2)$, $(a, 3)$ are indistinguishable in $M \mid\{Q\} .(M \mid\{Q\} / \varepsilon$ is isomorphic to $N$.

By Section $1 T_{i+1}$ is 5 -reducible to $T_{i}$ for every $0 \leqq i<5$ hence $T_{5}$ is 5 -reducible to $T_{0}$. By Section $8 T_{5}$ is 5 -inseparable hence $T_{0}$ is 5 -inseparable.

\section{Two Equivalence Relations}

Theorem 1. The theory of two equivalence relations is 5-inseparable.

Proof. Let $T_{0}$ be the theory of equivalence relations $A$ and $B$ and $T_{1}$ be the theory obtained from $T_{0}$ by introducing equality by means of the formula $A x y \wedge B x y$. Equivalence classes of $A$ (respectively $B$ ) will be called rows (respectively columns). Let $\alpha(x, y)$ [respectively $\beta(x, y)]$ abbreviate an existential formula saying that there is a column with at least 3 elements (respectively there are at least two columns) intersecting both the row of $x$ and the row of $y$. Let $T_{2}$ be the extension of $T_{1}$ by means of an axiom saying that if two different columns intersect each of two different rows then each of the columns comprises exactly 2 elements. $T_{2}$ is a 5-extension of $T_{1}$ and the formula

$$
\sim A x y \rightarrow \sim(\alpha(x, y) \wedge \beta(x, y))
$$

is a theorem of $T_{2}$. Let $T_{3}$ be the extension of $T_{2}$ by the axiom

$$
\sim A x y \rightarrow \alpha(x, y) \vee \beta(x, y) .
$$

Let $T_{3}$ be the theory obtained from $T_{2}$ by introducing a binary predicate $P$ by the formula

$$
\sim A x y \wedge \beta(x, y),
$$

which is neutral in $T_{2}$ for its negation is equivalent in $T_{2}$ to

$$
\sim A x y \rightarrow \alpha(x, y) .
$$

Let $T_{4}$ be the theory of an irreflexive symmetrical predicate $P$. We prove that $T_{4}$ is embedded into $T_{3} . L\left(T_{4}\right) \subseteq L\left(T_{3}\right)$ and the reduction of $T_{3}$ to the language $\{P$ \} extends $T_{4}$. Let $N$ be a finite economical model of $T_{4}$. We're looking for a finite model $M$ of $T_{3}$ such that the economical version of $M \mid\{P\}$ is isomorphic to $N$. The idea of constructing $M$ is simple. Put elements of $N$ into different rows of a table. If Pab holds in $N$ add 4 auxiliary elements forming two columns intersecting the rows of $a$ and $b$. If $P a b$ fails in $N$ add 3 auxiliary elements forming one column intersecting the rows of $a$ and $b$. The only problem is that in the latter case we have only two rows for a column of 3 elements. Build a model $M$ for $L\left(T_{3}\right)$ as follows. $|M|$ is the union of sets

$$
\begin{gathered}
S=|N| \times\{1,2,3\}\left[\text { we write } a_{i} \text { for }(a, i)\right], \\
\left\{\left(a_{i}, b_{j}, k\right): N \models P a b \text { and } i, j \in\{1,2,3\} \text { and } k \in\{1,2\}\right\}, \\
\left\{\left(a_{i}, b\right): N \models \sim P a b \text { and } i \in\{1,2,3\}\right\} .
\end{gathered}
$$


$A$ is defined in terms of rows, which are $A$-equivalence classes of course. Each row contains exactly one element of $S$. Elements $\left(a_{i}, b_{j}, k\right)$ and $\left(a_{i}, b\right)$ are in the row of $a_{i}$.

$B$ is defined in terms of columns, which are $B$-equivalence classes of course. For each $a$ the three elements $\left(a_{1}, a\right),\left(a_{2}, a\right),\left(a_{3}, a\right)$ form a column. If $a \neq b$ and $P a b$ fails in $N$ then the six elements $\left(a_{i}, b\right),\left(b_{j}, a\right)$ form a column. If $P a b$ holds in $N$ then for every $i, j, k$ the two elements $\left(a_{i}, b_{j}, k\right)$ and $\left(b_{j}, a_{i}, k\right)$ form a column.

If $x$ is in the row of $a_{i}$ and $y$ is in the row of $b_{j}$ then $P x y$ holds iff $P a b$ holds in $N$.

It is easy to see that $M$ is a finite model of $T_{3}$, the elements $a_{1}, a_{2}, a_{3}$ are indistinguishable in $M \mid\{P\}$ and the economical version of $M \mid\{P\}$ is isomorphic to $N$.

By Section $9 T_{4}$ is 5-inseparable. By Section $1 T_{i+1}$ is 5 -reducible to $T_{i}$ for $0 \leqq i<4$. Hence $T_{0}$ is 5-inseparable.

\section{REFERENCES}

[1] Büchi, J.R.: Turing machines and the entscheidungsproblem. Math. Ann. 148, 201-213 (1962).

[2] Gurevich, Y.: Existential interpretation. Algebra and Logic 4, 71-85 (1965).

[3] Gurevich, Y.: New applications of the existential interpretation method. Abstracts of 1972 Moscow Conference in Math. Logic.

[4] Gurevich, Y.: Semi-conservative reduction. Arch. math. Logik 18, 23-25 (1976).

[5] Lewis, H.R.: Unsolvable classes of quantificational formulas. Addison-Wesley Publishing $\mathrm{Co}$. (to appear).

[6] Malcev, A.I.: Algorithms and recursive functions. Moscow: Nauka 1965.

[7] Muchnik, A.A.: Isomorphism of systems of recursively enumerable sets with effective properties. Trudy (Transactions) of Moscow Math. Soc. 7, 407-412 (1958).

[8] Smullyan, R.M.: Theory of formal systems. Ann. Math. Studies 47 (1961).

[9] Suranyi, J.: Reduktionstheorie des Entscheidungsproblems. Budapest, 1959.

Yuri Gurevich

Mathematics Dept., Ben-Gurion

University, Beer-Sheva, Israel

and

Computer Science Dept.,

University of Michigan

Ann. Arbor, MI 48109 